\title{
TESTES DE USABILIDADE DE UM CONTROLE REMOTO COM IDOSOS: APLICAÇÃO DE TESTES VIRTUAIS EM SOFTWARES CAD/CAE
}

\author{
USABILITY TESTING OF A REMOTE CONTROL WITH \\ OLDER ADULT USERS: APPLICATION OF VIRTUAL \\ TESTING IN CAD/CAE SOFTWARES
}

Mayara Ramos ${ }^{1}$, Alexandre Amorim dos Reis²

RESUMO: O envelhecimento populacional traz a necessidade de desenvolver produtos mais adequados às capacidades dessa população. Uma forma de progredir nesse sentido é a inclusão do público idoso em testes de usabilidade no processo de desenvolvimento de produtos. Essa inclusão pode ser feita com baixo custo e sem exposição do usuário por meio de testes virtuais. O objetivo neste estudo é desenvolver um procedimento para testes de usabilidade virtuais realizados no software SolidWorks que foque nos idosos, realizando um estudo com um controle remoto. Para isso foram feitos tais testes com esse público, sendo um convencional e um com usuários simulados virtualmente, comparando seus resultados. A conclusão indicou que, devido às limitações encontradas neste estudo, o teste virtual na plataforma desse software ainda não substitui os testes tradicionais.

PALAVRAS-CHAVE: Usabilidade; Métodos para Fatores Humanos; Modelamento Digital Humano; Idosos; Software CAD/CAE.

ABSTRACT: Population aging brings the need to develop products better suited to the capabilities of the population. One way of progressing towards this direction is the inclusion of older adults in usability testing during the process of product development. This is possible at low cost and without exposing the user, with use of virtual testing. This study aimed to develop a procedure for virtual usability tests made in the software SolidWorks with a focus on older adult users. For this purpose, two usability tests were conducted with these users: a traditional test and a test with simulated users, both results being compared, and having as object studied a remote control. Comparison of the test results indicated that, due to the limitations found, virtual test in SolidWorks was not able to replace traditional tests.

KEYWORDS: Usability; Methods for Human Factors; Digital Human Modeling; Older populations, CAD/CAE software.

\section{INTRODUÇÃO}

A idade é uma variável relevante às considerações de design na pesquisa e prática de fatores humanos, e essa importância está relacionada a três principais motivos: o crescimento constante da população de idosos em países desenvolvidos, o aumento da proporção desses sujeitos dentre os trabalhadores (work force) e as significativas diferenças físicas e cognitivas entre jovens e idosos advindas do envelhecimento que necessitam de considerações específicas de projeto (NICHOLS; ROGERS; FISK, 2006). O aceleramento do envelhecimento populacional e a mudança demográfica que isso causa terão uma grande influência no gerenciamento de inovações e desenvolvimento de produtos e serviços em todos os segmentos da indústria, que precisam ser adaptados ou desenvolvidos para atender às mudanças e necessidades da população em envelhecimento (KOHLBACHER; HERSTATT; SCHWEISFURTH, 2010). 
A mudança do cenário etário requer uma maior preocupação com o projeto de produtos para que idosos possam se beneficiar dos produtos de massa (off the shelf) adequadamente (NICHOLS; ROGERS; FISK, 2006). Fabricantes e consumidores podem ser beneficiados com o aumento desse crescente mercado orientado pelas necessidades especiais e habilidades do público idoso. A análise rigorosa dessas características durante os estágios iniciais do projeto podem gerar produtos melhor adequados aos idosos, oferecendo-lhes maior satisfação (EHMEN et al., 2012).

Apesar de existirem muitos recursos, ferramentas e técnicas que auxiliam no design inclusivo, se tem uma escassez de dados que ajudem em uma previsão quantitativa do desempenho que esses usuários com capacidades limitadas terão ao interagir com os recursos dos produtos (LANGDON et al., 2015). Testes ergonômicos de usabilidade de produtos com a inclusão do público idoso podem contribuir para a melhoria da integração desse público com os itens colocados no mercado e respeitar as suas limitações físicas causadas pelo avanço da idade. O maior número de testes também pode trazer o aumento de dados quantitativos e qualitativos disponíveis para compreender o desempenho dos usuários.

\section{Design voltado para o público idoso e usabilidade}

As capacidades e limitações da população idosa devem ser compreendidas e levadas em consideração no processo de design e na pesquisa de fatores humanos para garantir que esse segmento da população possa interagir com produtos e sistemas de maneira segura, eficiente e eficaz (NICHOLS; ROGERS; FISK, 2006). Com o avançar da idade, as habilidades psicomotoras, perceptivas e cognitivas decaem. Apesar disso, poucos são os esforços para o desenvolvimento de tecnologias que compensem esse declínio (HIGGINS; GLASGOW, 2012). Para o desenvolvimento de produtos funcionais, eficientes, seguros e atraentes que otimizem as habilidades ou compensem as limitações, é necessário considerar as mudanças advindas da idade nos sentidos e nas capacidades físicas (HAIGH, 1993).

A usabilidade serve como um indicador de quão intuitivo é utilizar um produto e é um dos atributos de qualidade perceptível aos usuários. Essa característica é determinante no sucesso de um produto e influencia diretamente o interesse do usuário em fazer seu uso (SILVA FILHO, 2010).

Um produto útil apresenta uma boa interação entre necessidade e solução, entre usuário e artefato. Avaliações de usabilidade são as abordagens utilizadas para garantir que um produto, sistema ou serviço sejam úteis. Esses testes podem ser realizados tanto para determinar se existem dificuldades de uso ou para descobrir oportunidades de melhorias (NEMETH, 2004).

A maior parte dos estudos que vêm sendo publicados sobre testes de usabilidade com idosos comparam os resultados de usuários idosos com jovens. Segundo Sonderegger, Schmutz e Sauer (2016), esses resultados mostram que, durante o uso de artefatos, o desempenho (eficiência e eficácia) dos idosos é geralmente mais baixo que o dos mais jovens. Ainda segundo os autores, nos estudos em que os artefatos foram reprojetados para aprimorar o desempenho dos idosos, os jovens também foram beneficiados.

\section{Testes virtuais e DHM}

Para se alcançar o desempenho total de produtos, muitas ferramentas e tecnologias de fatores humanos ou ergonomia têm evoluído com os anos para auxiliar antecipadamente análises e design. Tal como em muitas áreas da Engenharia, um excelente candidato para essa interação é a modelagem computacional e simulação (SUNDIN; ÖRTENGREN, 2006). A modelagem computacional é bastante difundida com o uso de softwares CAD (Computer Aided Design) e CAE (Computer Aided Engineering), já a simulação nos fatores humanos tem sido aplicada no Digital Human Modeling (DHM). 
DHM representa a tecnologia de usar o computador para construir uma representação virtual de uma pessoa para simular movimento e esforços humanos (CHAFFIN et al., 2001). Esses softwares, em sua maioria, modelam ergonomia física (THORVALD; HÖBERG; CASE, 2009) e objetivam promover suporte antropométrico: análises, medidas e avaliações no design do produto e sua produção, analisando virtualmente alcance, visão etc., antes que qualquer objeto físico seja construído (JUNG et al., 2009; SUNDIN; ÖRTENGREN, 2006). Ferramentas de DHM permitem verificar se a tarefa a ser executada com o artefato projetado é aceitável ergonomicamente ou não por prever e analisar quão bem as dimensões de um produto ou sistema produtivo irão adaptar-se às dimensões corporais do futuro usuário e a interação dele com o produto (SUNDIN; ÖRTENGREN, 2006). Essas análises também permitem otimizar os locais de trabalho e minimizar o risco de lesões musculoesqueléticas relacionadas com o trabalho (LMERT), como aquelas decorrentes de posturas incômodas e uso de força excessiva (CHANDER; CAVATORTA, 2018).

A incorporação de um modelo digital que simula as ações humanas no processo de projeto de produtos tem sido reconhecida como eficaz no desenvolvimento do design de produtos centrados no usuário. O humano digital também pode apresentar informações úteis como biotipo, dimensões, restrições de movimento e sequências de operação em um espaço 3D que devem ser levados em consideração no detalhamento do design. Permitir ao usuário final, representado pelo manequim, interagir com o modelo do produto facilita sua personalização à medida que o projeto se desenvolve (KUO; CHU, 2005).

Apesar de que estudos tenham contribuído muito para o desenvolvimento dos modelos digitais humanos, métodos adequados ao modelamento humano para sistemas CAD ainda não existem em relação aos aspectos de precisão dimensional e confiabilidade dos resultados (BAEK; LEE, 2012). As bases de dados que são usadas para a construção dos modelos digitais humanos são muito limitadas quando se trata de pessoas que fogem do padrão, como é o caso dos idosos (PORTER et al, 2004).

Ainda não foram desenvolvidos modelos científicos de avaliação de usabilidade para softwares DHM. A avaliação de usabilidade nessas ferramentas ocorre da relação entre medidas subjetivas e índices objetivos. As medidas subjetivas podem ser avaliadas por uma combinação ponderada de limitações angulares de articulações e avaliação de alcance (Mochimaru, 2017). Barbé et al. (2012), por exemplo, fazem uma análise de desempenho da tarefa por meio de análises de precisão dos gestos, duração e dificuldade da tarefa usando um software DHM, nesse caso, um estudo de telas sensíveis ao toque em cockpit de aeronaves. A análise de usabilidade também é utilizada como apoio para as avaliações ergonômicas realizadas nesses softwares, por essa análise são identificados quais os pontos em que os usuários apresentam maior dificuldade no produto analisado (GIRONIMO et al., 2012).

\section{Problemática e objetivo do estudo}

Como foi apontada, a mudança do cenário etário mundial requer uma maior preocupação nos projetos de produtos para que os idosos possam usufruir das opções existentes no mercado de forma adequada. A inclusão dessa população em testes de usabilidade dos produtos acaba trazendo benefícios também para os demais usuários, já que a interação com os artefatos é simplificada.

O uso de softwares de DHM permite que os testes virtuais dos produtos e sistemas sejam realizados em etapas antecipadas do projeto de forma mais rápida e com menor custo, além de não apresentar riscos para os sujeitos por utilizar usuários virtuais/simulados. No entanto, esses programas ainda possuem falhas em compatibilidade com softwares CAD/CAE, além de as empresas necessitarem da aquisição de licença para mais uma ferramenta de projeto. Outros problemas enfrentados no uso desses softwares são a falta 
de dados de usuários como as dos idosos, que apresentam algumas de suas habilidades debilitadas devido ao processo de envelhecimento, e ausência de protocolos específicos para análise de usabilidade.

Considerando esse cenário, este trabalho apresenta um estudo inicial de uma proposta de análise de usabilidade de interações físicas, com usuários idosos, realizado em uma plataforma virtual de um software CAD/CAE. Para isso foi desenvolvido e avaliado um procedimento para testes de usabilidade virtuais realizados no software SolidWorks, com foco no usuário idoso. Com esse objetivo foi reproduzido um teste de usabilidade convencional de um controle remoto, realizado com usuários idosos (60 a 85 anos), em um teste de usabilidade virtual na plataforma do SolidWorks, em que o usuário modelado virtualmente apresenta limitações compatíveis às dos usuários participantes do teste convencional, podendo assim comparar os resultados encontrados nos testes.

Para realizar o teste virtual no SolidWorks, trabalhou-se com a hipótese de que o software apresenta ferramentas de simulação que podem ser utilizadas para execução de testes virtuais de usabilidade realizados diretamente na sua plataforma, tendo foco no público idoso, substituindo testes de usabilidade convencionais. Esse software é comumente utilizado no desenvolvimento de projetos de produtos e no ensino superior de cursos relacionados a essa área. A realização desses testes em softwares de modelagem e simulação CAD/CAE pode melhorar a aplicação dessas investigações, pois os usuários desses programas não necessitarão de um treinamento para interagir com uma nova plataforma.

\section{METODOLOGIA}

\section{Indivíduos do estudo}

Participaram do estudo 25 idosos, 12 homens e 13 mulheres, com idade entre 60 e 85 anos (média=68,19). Os participantes dessa pesquisa não apresentam registros de doenças musculoesqueléticas, neurológicas graves ou baixa acuidade e possuem controle sobre outras possíveis doenças, como diabetes e hipertensão. Esse perfil de usuário foi escolhido para evitar grandes desvios de resultados entre os usuários. Os participantes foram recrutados no Grupo de Estudos da Terceira Idade (Geti) da Universidade do Estado de Santa Catarina (Udesc) e aceitaram voluntariamente participar do estudo, assinando um Termo de Consentimento Livre Esclarecido (TCLE).

\section{Teste de usabilidade e métricas do estudo}

Os testes realizados nesse estudo se deram em três etapas: coleta de dados antropométricos e realização do teste convencional de usabilidade; modelamento e realização dos testes em plataforma virtual; e, por fim, comparação dos resultados dos testes - o convencional e o realizado em plataforma virtual.

A atividade realizada pelos sujeitos pesquisados no teste de usabilidade foi a de pressionar teclas determinadas de um controle remoto de televisão segurando o objeto da forma que o usuário considerasse a mais confortável. A Figura 1 mostra uma representação do controle remoto utilizado. O uso desse produto escolhido para o teste se deu em razão dos resultados da pesquisa de Strehlau, Bacha e Lora (2006), que tinha como objetivo identificar quais atividades de lazer as pessoas da terceira idade se dedicam com maior frequência e agrupá-las segundo faixa etária, renda média e gasto médio; dos 700 participantes com 60 anos ou mais, todos declararam assistir à televisão como uma das atividades de lazer mais frequentes. 


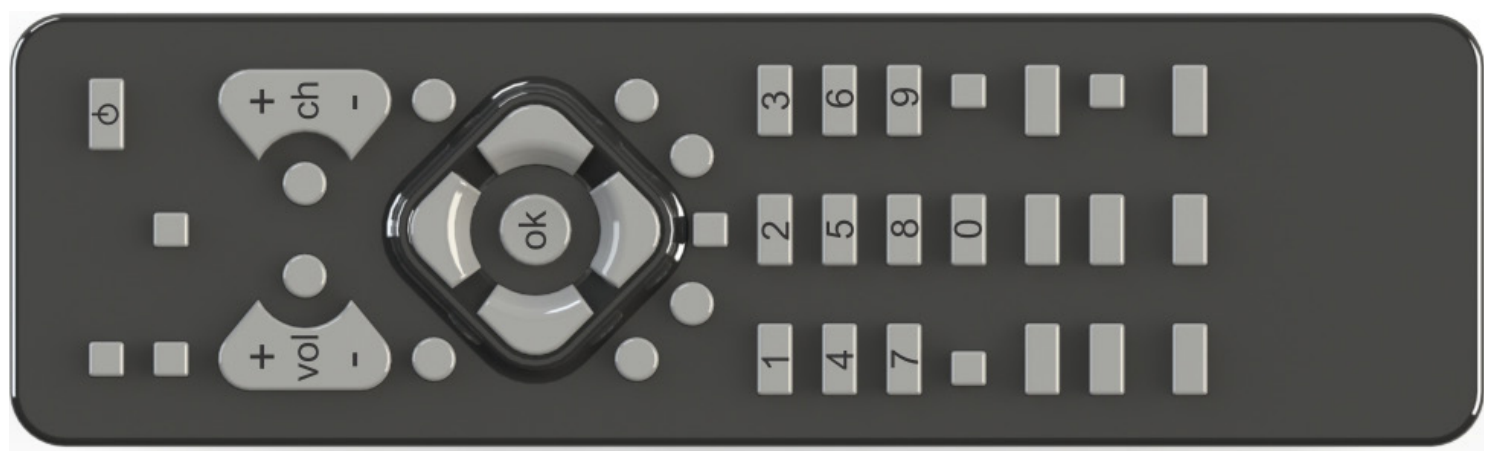

Nesse teste foram medidos o sucesso da tarefa e o número de reposicionamentos necessários para efetuá-la. Este último dado se dá pela quantidade de vezes que o indivíduo muda o local de apoio do controle remoto na mão. Na métrica do sucesso da tarefa, o foco é voltado para a limitação de movimentos que ocorre com o envelhecer e como isso pode afetar o acionamento de botões/comandos, assim como a indesejável possibilidade de se apertar mais de um botão simultaneamente. O sucesso da tarefa se dá pelo pressionamento apenas das teclas indicadas. Caso o indivíduo tenha realizado a tarefa com auxílio do mediador, foi considerada a realização parcial da tarefa (sucesso parcial com auxílio). Quando o indivíduo, ao realizar a tarefa tenha cometido erros, como acionar um botão que não correspondia ao solicitado, a tarefa foi tida como de realização parcial com erros. O indivíduo que não conseguiu realizar a tarefa pressionando apenas o botão solicitado e acionando mais de um botão, ou no caso dos botões de canal e volume, nos quais o mesmo botão é dividido em duas regiões, para aumentar ou diminuir, e se for pressionada no meio do botão (região neutra), a tarefa foi considerada como não realizada com sucesso.

As métricas avaliadas na comparação dos resultados dos testes convencional e virtual foram o sucesso da tarefa e número de reposicionamentos do objeto de análise para completar a tarefa. Para a análise do teste de usabilidade convencional utilizou-se da observação para obtenção dos dados. Para o teste virtual foi executada uma experimentação ao se simular o teste realizado anteriormente de forma convencional para comparar esses dados alcançados e, assim, procurar a corroboração da hipótese de que os resultados desses dois tipos de testes não apresentam diferenças estatísticas significativas.

Este trabalho passou por uma avaliação de Comitê de Ética e foi aprovado sob o Número CAAE 21379913.2.0000.0118.

\section{Materiais}

Para a realização do estudo foram utilizados controle remoto de grande presença no mercado local, câmera fotográfica e filmadora em tripés, retículo acrílico quadriculado com espaçamento de $5 \mathrm{~mm}$ entre as linhas para calibração da imagem, estrutura para manter o retículo perpendicular à superfície de apoio, paquímetro de $150 \mathrm{~mm}$ e goniômetro para a coleta de dados. Para o teste virtual foram utilizados os softwares Kinovea para obtenção das medidas das mãos (software livre) e SolidWorks 2014 licenciado para a Universidade do Estado de Santa Catarina.

\section{Procedimento de coleta de dados}

Os dados deste trabalho foram coletados em dois momentos, teste de usabilidade convencional, seguido da medição de antropometrias com os indivíduos idosos, e a modelagem das antropometrias e execução do teste de forma virtual na plataforma do software SolidWorks.
Figura 1: Modelo do controle remoto

Fonte: elaborado pelos autores 
Figura 2: Configuração para teste de usabilidade

Fonte: Elaborado pelos autores
Para o primeiro momento de coleta de dados, foi adotado o seguinte protocolo: os participantes assinaram o TCLE e foi preenchida uma ficha com dados cadastrais do participante (idade, sexo, membro dominante), após isso o participante foi acomodado na posição sentada, ficando de lado para a câmera filmadora (Figura 2). O objeto do estudo foi apresentado aos participantes visando a redução do número de erros; solicitou-se o acionamento de uma sequência de botões (ligar/desligar, 2, 5, 0, ok, ch+, vol-), que foi registrada em vídeo. Após o término da atividade foi aplicado um questionário pós-teste sobre a percepção da atividade.
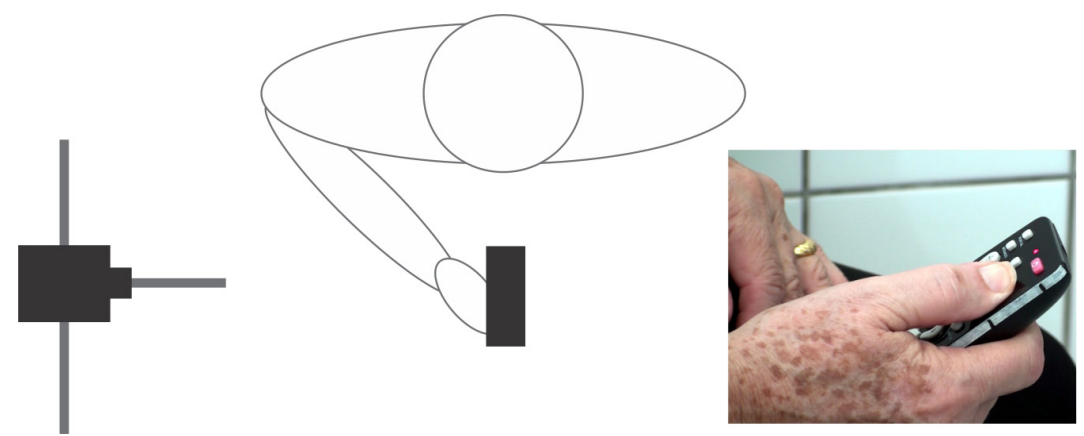

Para a mensuração das antropometrias, foram destacados com caneta hidrográfica preta os pontos onde se localizam as articulações dos dedos. A mão foi posicionada de forma aberta, com os dedos juntos sobre o retículo acrílico, que mantém o sistema calibrado. A câmera fotográfica foi posicionada perpendicularmente à área palmar sendo fotografada (Figura 3). O mesmo foi realizado para a mão espalmada. Por fim, foram medidas com o goniômetro as flexões máximas das articulações interfalangianas do polegar, e com o paquímetro foram mensurados a ponta do polegar, o diâmetro das articulações dos polegares e a espessura da mão.
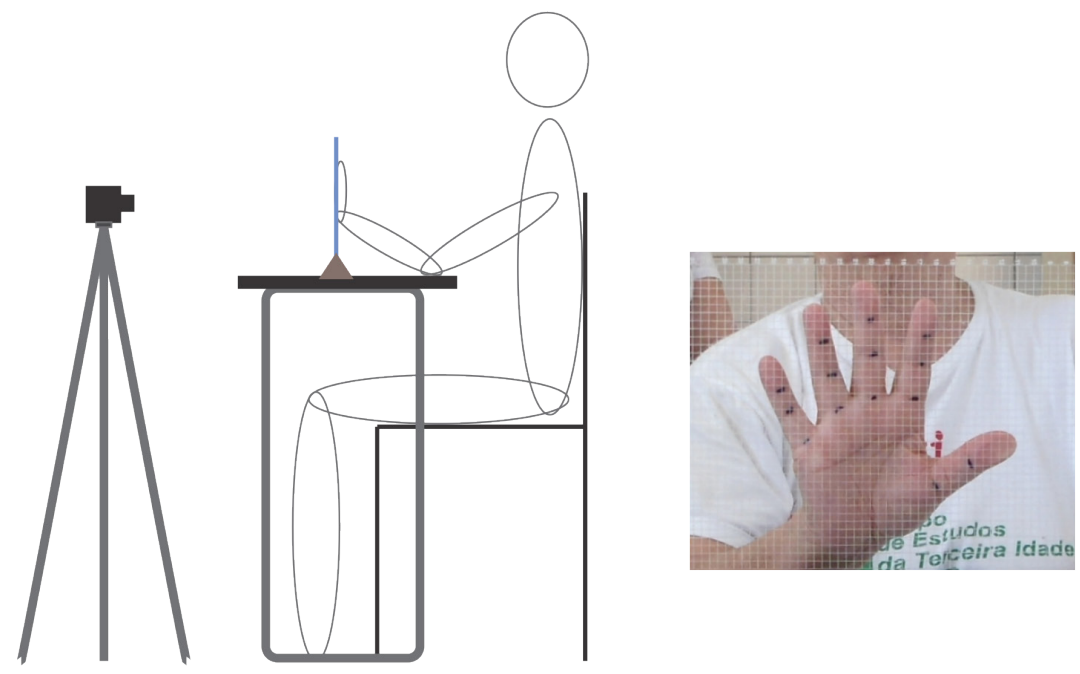

A segunda etapa consistiu no modelamento virtual das antropometrias em plataforma CAD (SolidWorks). As medidas dos polegares dos idosos foram modeladas no software citado, e foi criado um modelo virtual para a simulação da tarefa. Para a modelagem do metacarpo e das falanges proximal e distal do polegar, foram utilizados as medidas da espessura da mão, o diâmetro das articulações interfalangianas proximal e distal, as medidas da ponta do polegar e o comprimento dos segmentos que compõem o polegar para dimensionar os 
seus respectivos componentes simulados na modelagem. Os comprimentos dos segmentos do polegar foram obtidos pelas fotografias das mãos por meio do software Kinovea. Para a modelagem foi utilizado o recurso de tabela de projeto para que cada configuração derivada representasse as antropometrias de um dos indivíduos participantes do teste real. A Figura 4 ilustra a modelagem dos segmentos do polegar (metacarpo, falanges proximal e distal).

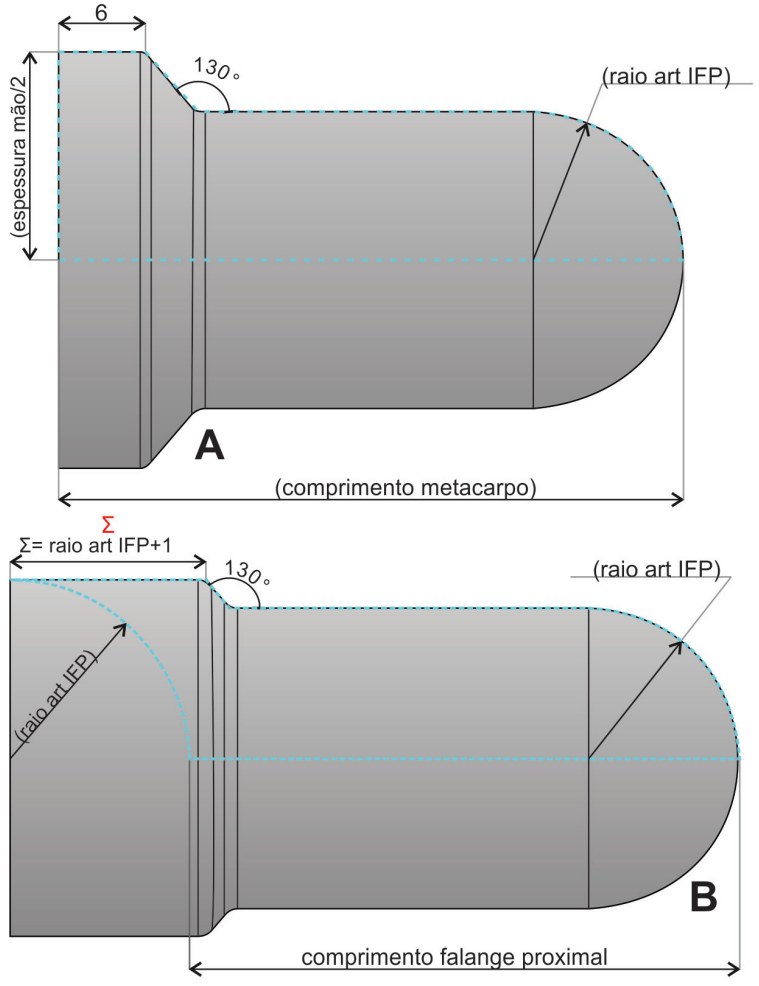

Com a modelagem de uma réplica do controle utilizado nos testes convencionais e o modelamento aproximado do polegar dos indivíduos idosos, foi elaborado um arquivo de montagem no programa SolidWorks. $\mathrm{O}$ controle é posicionado de forma fixa, com a origem da peça coincidente com a do arquivo de montagem alinhando os planos. Na junção do metacarpo e da falange proximal e entre flanges proximal e distal foram efetuados posicionamentos concêntricos e posicionamento avançado de angulação entre os planos superiores, que atribuíram uma angulação mínima e máxima para cada indivíduo, representando uma faixa de flexão de conforto.

Com a análise do comportamento de uso do controle e as medidas das flexões máximas dos indivíduos, determinou-se a faixa de flexão de conforto para as articulações interfalangianas proximal e distal de cada indivíduo. Para determinar as faixas de conforto, foram diminuídos $3^{\circ}$ dos limites (máximo e mínimo), sendo a flexão mínima ou de repouso para a articulação interfalangiana proximal de $3^{\circ}$ (seguindo o apontado por Settineri (1988) para a extensão ativa de $0^{\circ}$ ) e a máxima se dá pela medida tomada com o goniômetro no usuário $-3^{\circ}$. 0 mesmo se aplica para a flexão máxima da articulação interfalangiana distal, sendo que flexão de repouso é de $8^{\circ}\left(5^{\circ}\right.$ indicado por Settineri (1988), $+3^{\circ}$ ).

Foram realizados posicionamentos para impossibilitar que algumas posições fossem adotadas pelo modelo de polegar. Essas posturas foram restringidas por serem posições que o usuário não conseguiria adotar ou seriam desconfortáveis. A primeira restrição foi um posicionamento avançado de
Figura 4: Modelagem dos segmentos metacarpo, falange proximal e falange distal

Fonte: Elaborado pelos autores 
Figura 5: Restrições de movimento - simulação

Fonte: elaborado pelos autores angulação entre o plano frontal das falanges e a face da superfície do controle remoto, sendo a angulação mínima de $15^{\circ}$ e a máxima de $90^{\circ}$ (perpendicular). A próxima, um posicionamento avançado de distância entre a face da superfície da interface do controle com o ponto central da base do metacarpo, a distância mínima foi de 12,5 mm (raio da maior articulação metacarpiana medida nos indivíduos), e para distância máxima adotada foi o comprimento do polegar multiplicado pelo seno de $30^{\circ}$, correspondendo à altura que o ponto central da base do metacarpo ficaria com o dedo estendido em uma angulação de $30^{\circ}$ relativamente ao controle. A Figura 5 ilustra essas restrições de posicionamento aplicado.

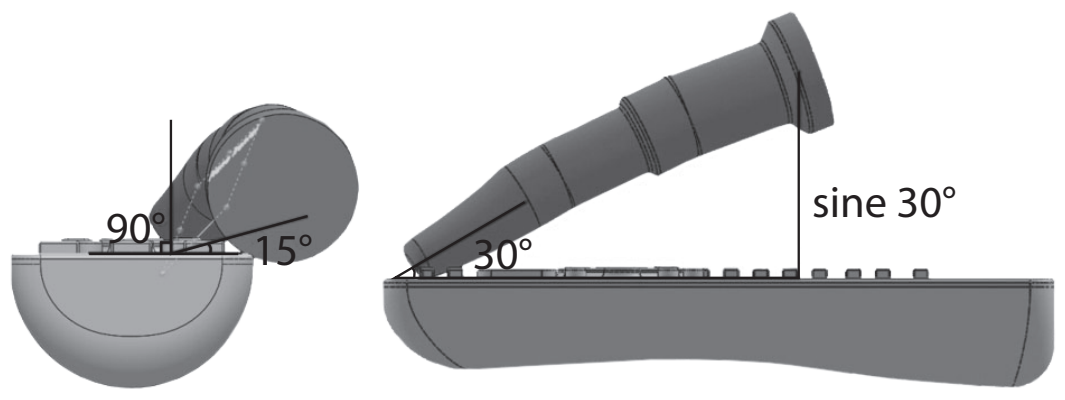

Para simular a atividade foi feito um posicionamento tangencial entre a ponta da falange distal e a superfície do controle, e para o acionamento de cada botão é feito um posicionamento de coincidência entre o ponto central do botão e a face da ponta da falange distal. Esse posicionamento de coincidência é editado para cada botão correspondente aos utilizados no teste convencional. O posicionamento inicial para cada modelo simulado de um participante pesquisado é no botão ligar com as falanges esticadas ao máximo, dentro das limitações impostas.

\section{Análise de dados}

As medidas das mãos foram tomadas por meio das imagens coletadas com o uso do software Kinovea 0.8.15, software gratuito, que, a partir de uma medida conhecida (retículo quadriculado), permite calibrar a imagem e obter as outras medidas. Filmou-se o teste de usabilidade realizado pelos indivíduos estudados e, pela observação do vídeo, foram analisados o sucesso da tarefa e o número de reposicionamentos necessários para realizá-la.

As demais etapas do experimento foram executadas no software SolidWorks. Na simulação do número de reposicionamentos necessários para realizar a tarefa, estipulou-se que, caso a ponta do polegar fique abaixo da base do metacarpo, é necessário um novo posicionamento. Isso foi definido, pois essa posição provavelmente não seria adotada pelo sujeito real por gerar desconforto ou limitação física do conjunto de segmentos que formam a mão. O reposicionamento também é efetuado caso a ponta do polegar não alcance o botão determinado.

\section{Tratamento estatístico}

Na comparação entre os resultados do teste convencional com idosos e o teste realizado na plataforma SolidWorks, utilizou-se o teste estatístico de Wilcoxon pareado. Essas comparações foram divididas por sexo e então comparadas entre os resultados dos testes convencional e simulado. O nível de significância empregado foi de $5 \%(\alpha=0,05)$, procurando um nível de confiabilidade dos dados de $95 \%$. Os testes estatísticos foram realizados no software IBM SPSS Statistics licenciado para Universidade do Estado de Santa Catarina. 


\section{RESULTADOS}

\section{Sucesso da tarefa}

No teste convencional de usabilidade 13 participantes obtiveram sucesso ao realizar a tarefa (8 mulheres, 5 homens), 6 realizaram a tarefa parcialmente com ajuda do moderador (3 mulheres e 3 homens) e 4 alcançaram um resultado parcial com erros ( 2 mulheres, 2 homens), enquanto 2 homens não completaram a tarefa. No teste virtual com usuários simulados, 23 concluíram a tarefa com sucesso (12 mulheres, 11 homens) e 2 não completaram a tarefa (1 mulher e 1 homem). 0 Gráfico 1 mostra ilustra esses resultados.

Gráfico 1: Sucesso da tarefa

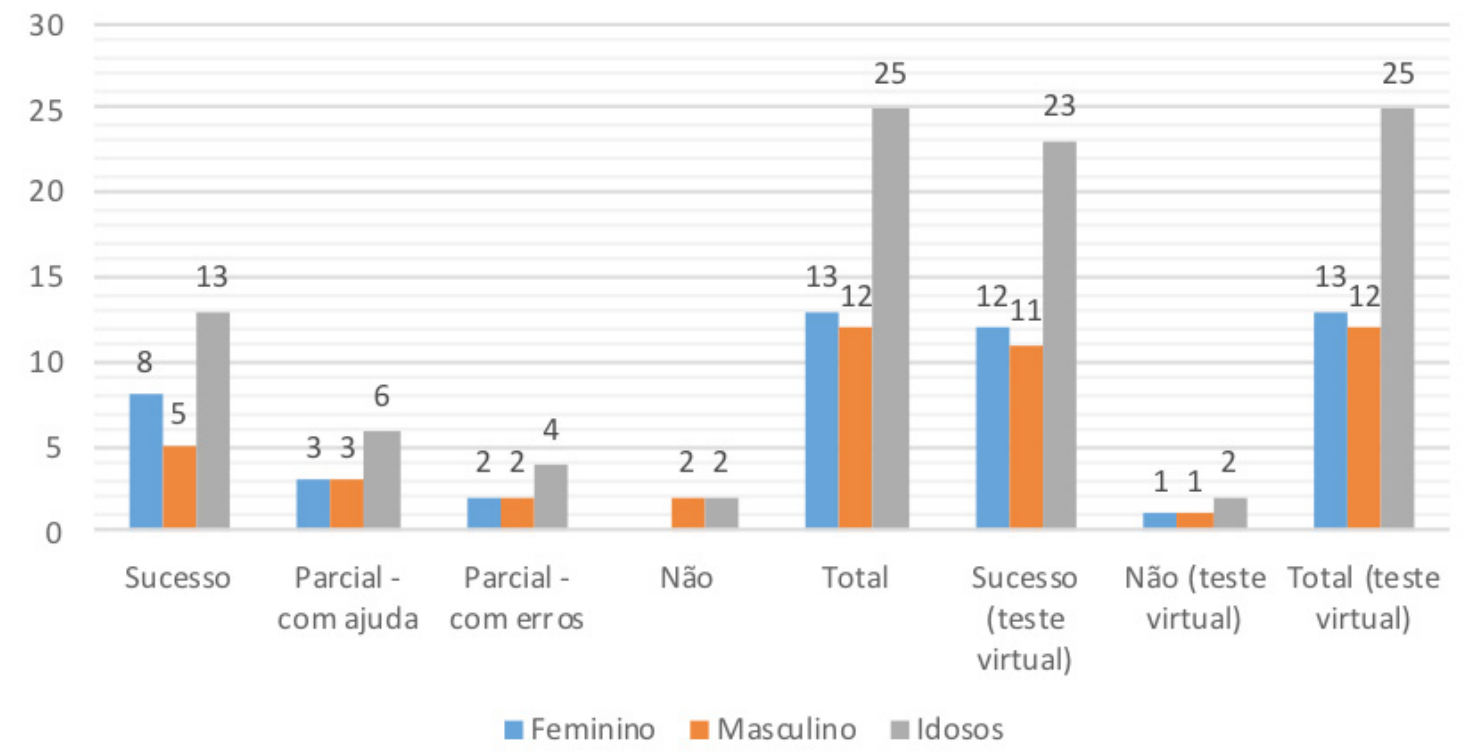

Fonte: elaborado pelos autores

Para comparar os resultados encontrados pelos testes convencional e virtual, utilizou-se o teste de hipótese de Wilcoxon. Para essa análise, os dados de sucesso parcial (com auxílio e com erros) no teste convencional foram considerados como sucesso da tarefa, pois no teste virtual não foi possível simular as questões cognitivas que levam ao resultado de sucesso parcial. O teste indicou que não existe uma diferença significativa dos resultados encontrados para sucesso da tarefa no teste convencional comparado ao virtual, o valor de "p" encontrado foi de 0,564 . No tratamento estatístico quando realizado agrupando os idosos por sexo, o valor encontrado no teste para mulheres idosas foi de $p=0,317$ e para homens idosos, $\mathrm{p}=1$. Isso sugere que os homens idosos apresentam resultados tendendo a igualdade entre os métodos e maior similaridade estatística do que as mulheres idosas.

\section{Número de reposicionamentos para completar a tarefa}

No teste convencional houve sujeitos que realizaram a tarefa sem reposicionamentos do objeto e sujeitos que realizaram até 6 , tendo uma mediana de 2 reposicionamentos. No teste virtual a variação entre o número máximo e mínimo de reposicionamentos é menor, sendo o mínimo zero e o máximo 2. A mediana nesse caso é de nenhum reposicionamento do objeto. A Tabela 1 apresenta os resultados dos testes agrupados por sexo. 
Tabela 1: Resultados do número de reposicionamentos agrupados por sexo

\begin{tabular}{l|c|c|c|c|c|c|c|}
\hline \multicolumn{1}{c}{ № de reposicionamentos } & Mínimo & Máximo & Média & Mediana & DP* & IC** Iower & IC upper \\
\hline Homens (convencional) & 1 & 6 & 2,67 & 2 & 1,43 & 1,75 & 3,58 \\
\hline Mulheres (convencional) & 0 & 6 & 1,77 & 1 & 1,69 & 0,75 & 2,79 \\
\hline Teste convencional $(H+M)$ & 0 & 6 & 2,20 & 2 & 1,60 & 1,54 & 2,86 \\
\hline Homens (virtual) & 0 & 2 & 0,33 & 0 & 0,77 & $-0,16$ & 0,83 \\
\hline Mulheres (virtual) & 0 & 2 & 1,23 & 2 & 1,01 & 0,62 & 1,84 \\
\hline Teste virtual (H+M) & 0 & 2 & 0,80 & 0 & 1,00 & 0,39 & 1,21 \\
\hline
\end{tabular}

Fonte: elaborado pelos autores

${ }^{(*)} \mathrm{DP}$ : desvio padrão.

${ }^{(+*)}$ IC: índice de confiança (inferior e superior).

Na comparação dessa variável entre os testes convencional e virtual também foi utilizado o teste de Wilcoxon. O resultado para o grupo dos usuários idosos foi $\mathrm{p}=0,002$, apontando para a existência de diferença significativa dessa medida entre os testes. Também aplicou-se o teste de Wilcoxon com os dados dos grupos idosos pareados por sexo. O resultado encontrado para os homens está condizente ao encontrado para o grupo, indicando que há diferença significativa entre os testes real e virtual $(p=0,004)$. Para as mulheres idosas o resultado foi $\mathrm{p}=0,271$, adotando-se a hipótese de que não existe diferença significativa de reposicionamentos para realização da tarefa entre o teste real e virtual.

\section{DISCUSSÃO - CONCLUSÃO}

Este trabalho foi motivado pelo envelhecimento populacional, pela necessidade da adequação de projetos e produtos para esses usuários e pela possibilidade de os meios virtuais de avaliação ergonômica e usabilidade poderem ser uma forma rápida e de baixo custo no desenvolvimento de produtos. Com isso, procurou-se elaborar e avaliar um procedimento para testes de usabilidade virtuais de idosos, realizados em um software de desenvolvimento de projetos de amplo uso na área de Design de Produtos (SolidWorks).

Os dados antropométricos e a análise do uso do controle remoto permitiram a modelagem e configuração dos movimentos na plataforma do SolidWorks de acordo com as características dos idosos para realizar os testes virtuais nessa plataforma. Para a configuração dos movimentos e as limitações, foram usadas as opções de posicionamento dos arquivos de montagem do SolidWorks.

Avaliaram-se nos testes convencional e virtual as métricas de sucesso da tarefa e o número de reposicionamentos necessários para sua realização. A métrica de sucesso da tarefa não apresentou diferenças estatísticas significativas entre os resultados. No entanto, essa é uma métrica que pode ter relações com a cognição, o que, ao considerarmos os resultados de sucesso parcial como sucesso da tarefa para a comparação dos testes, pode ter levado a essa resolução. A atividade avaliada se mantém constante no cotidiano dos sujeitos, sendo menos influenciada pelo envelhecimento, portanto, considerando a afirmação de Spirduso (2005) de que as perdas de força quase não são perceptíveis nos músculos que permanecem ativos, o mesmo poderia ser aplicado a uma atividade que se manteve presente ao longo da vida. Por esse motivo, essa variável não foi considerada como a ideal para avaliar a execução de testes virtuais de usabilidade pelo SolidWorks.

A avaliação do número de reposicionamentos para realizar a tarefa apontou para uma diferença significativa dos resultados, o que leva a conclusão de que a avaliação ergonômica virtual da usabilidade do controle remoto na plataforma do SolidWorks, no momento, não substitui o teste tradicional, ao menos com o modelo proposto neste trabalho. No entanto, essa mesma variável, 
quando avaliada apenas com as mulheres idosas, aponta um resultado em que não há uma diferença significativa entre o teste realizado na plataforma do software SolidWorks e o tradicional. Defende-se que, com a continuidade de estudos para o aprimoramento do método de avaliação na plataforma do software, esse resultado pode atingir também outros grupos de usuários.

O comportamento humano é uma limitação nas ferramentas de simulação de uso. No caso deste estudo, não foi possível inserir componentes cognitivos à simulação. Os participantes idosos apresentaram posturas de uso variadas ao executar uma mesma tarefa, cada usuário adota a postura de sua preferência, que julga ser a mais confortável, e essas escolhas pessoais não podem ser simuladas. Por ter-se adotado um padrão para a simulação, essa questão de preferências pessoais pode influenciar na comparação dos resultados do teste virtual e do tradicional, tanto para a métrica de sucesso da tarefa quanto para o número de reposicionamentos necessários para realizá-la.

A forma como os dados foram coletados poderia ter auxiliado no estudo do comportamento de uso dos indivíduos pesquisados. Se durante a realização da tarefa tivessem sido usados sensores ou acelerômetros, poder-se-iam obter dados que especificassem os ângulos que o dedo de acionamento adotou durante a atividade. As limitações encontradas neste estudo ocorreram em parte por ser uma pesquisa inicial na área de análise ergonômica em um software $\mathrm{CAD} / \mathrm{CAE}$, e em trabalhos futuros dessa área será possível corrigir essas limitações para se obterem melhores resultados.

Para possibilitar que o teste virtual de usabilidade na plataforma do SolidWorks tenha resultados similares ao teste convencional, sugere-se que em estudos futuros sejam coletados dados de um número maior de sujeitos para alcançar um estudo amplo do comportamento do uso do controle remoto. Dentre os dados coletados é importante controlar a angulação exata que o dedo de acionamento adota ao executar cada movimento no botão durante a tarefa, para então poder reproduzir esse comportamento na simulação do teste. Também sugere-se que se faça a mensuração das mãos com uso de scanner 3D, tendo dados mais próximos ao real, bem como medir a força e amplitude de movimento de todas as articulações da mão. Com esses dados é possível modelar toda a mão dos indivíduos e replicar com maior eficácia o desempenho dos usuários na plataforma do SolidWorks.

\section{AGRADECIMENTOS}

Os agradecimentos às instituições de apoio e fomento à pesquisa: Coordenação de Aperfeiçoamento de Pessoal de Nível Superior (Capes) e ao Conselho Nacional de Desenvolvimento Científico e Tecnológico (CNPq).

\section{REFERÊNCIAS}

BAEK, S. Y.; LEE, K. Parametric human body shape modeling framework for humancentered product design. Computer-Aided Design, Amsterdam, v. 44, n. 1, p. 56-67, 2012.

BARBÉ, J. et al. Physical ergonomics approach for touch screen interaction in an aircraft cockpit. In: CONFERENCE ON ERGONOMIE ET INTERACTION HOMME MACHINE, 17-19 out. 2012, Biarritz. Anais... New York: ACM Press, 2012.

CHAFFIN, D. B. et al. Digital human modeling for vehicle and workplace design. Pittsburgh: Society of Automotive Engineers, 2001.

CHANDER, D. S.; CAVATORTA, M. P. Multidirectional one-handed strength assessments using AnyBody Modeling Systems. Applied Ergonomics, Amsterdam, v. 67, p. 225-236, 2018.

EHMEN, H. et al. Comparison of four different mobile devices for measuring heart rate and ECG with respect to aspects of usability and acceptance by older people. Applied Ergonomics, Amsterdam, v. 43, n. 3, p. 582-587, 2012.

SILVA FILHO, A. M. Avaliação de usabilidade: "Separando o joio do trigo". Revista Espaço Acadêmico, Maringá, n. 112, p. 10-14, 2010.

GIRONIMO, G. et al. A top-down approach for virtual redesign and ergonomic optimization of an agricultural tractor's driver cab. In: BIENNIAL CONFERENCE ON ENGINEERING 
SYSTEMS DESIGN AND ANALYSIS, 11., 02-04 jul. 2012, Nantes. Anais... New York: ASME, 2012.

HAIGH, R. The ageing process: a challenge for design. Applied Ergonomics, Amsterdam, v. 24, n. 1, p. 9-14, 1993.

HIGGINS, P. G.; GLASGOW, A. Development of guidelines for designing appliances for older persons. A Journal of Prevention, Assessment \& Rehabilitation, Amsterdam, v. 41, n. 1, p. 333-339, 2012.

JUNG, M. et al. Integrated framework for vehicle interior design using digital human model. Journal of Computer Science and Technology, New York, v. 24, n. 6, p. 1149-1161, 2009.

KOHLBACHER, F;HERSTATT, C:SCHWEISFURTH, T. Product development for the silver market. In: KOHLBACHER, F.; HERSTATT, C. The Silver Market Phenomenon: marketing and innovation in the aging society. 2. ed. New York: Springer, 2010. p. 3-13.

$\mathrm{KUO}, \mathrm{C}$. F.; CHU, C. H. An online ergonomic evaluator for 3D product design. Computers in Industry, Amsterdam, v. 56, n. 5, p. 479-492, 2005

LANGDON, P. et al. A framework for collecting inclusive design data for the UK population. Applied Ergonomics, Amsterdam, v. 46, p. 318-24, 2015.

MOCHIMARU, M. Digital human models for human-centered design. Journal of Robotics and Mechatronics, Tokyo, v. 29, n. 5 , p. 783-789, 2017

NEMETH, C. P. Human factors methods for design: making systems human-centered. Boca Raton: CRC Press, 2004
NICHOLS, T. A.; ROGERS, W. A.; FISK, A. D. Design for Aging. In: SALVENDY, G. Handbook of human factors and ergonomics. Hoboken: John Wiley \& Sons, 2006. p. 1418-1445.

PORTER, J. M. et al. "Beyond Jack and Jill": designing for individuals using HADRIAN. International Journal of Industrial Ergonomics, Amsterdam, v. 33, n. 3, p. 249-264, 2004.

SETTINERI, L. I. C. Biomecânica: noções gerais. São Paulo: Livraria Atheneu, 1988.

SPIRDUSO, W. W. Dimensões físicas do envelhecimento. Barueri: Manole, 2005.

SONDEREGGER, A.; SCHMUTZ, S.; SAUER, $J$. The influence of age in usability testing. Applied Ergonomics, Amsterdam, v. 52, p. 291-300, 2016

STREHLAU, V.; BACHA, M.; LORA, M. Idosos não são iguais: uma análise de agrupamentos sobre as atividades de lazer da terceira idade. In: ENCONTRO DE MARKETING DA ANPAD, 2., 3-5 maio 2006, Rio de Janeiro. Anais..., Rio de Janeiro: Gráfica Editora Palotti, 2006.

SUNDIN, A.; ÖRTENGREN, R. Digital human modeling for CAE applications. In: SALVENDY, G. Handbook of human factors and ergonomics. Hoboken: John Wiley \& Sons, 2006. p. 1053-1078.

THORVALD, P.; HÖGBERG, D.; CASE, K. Incorporating cognitive aspects in digital human modeling. In: INTERNATIONAL CONFERENCE ON DIGITAL HUMAN MODELING - HCII, 13., 19-24 jul. 2009, San Diego. Anais... Berlin: Springer, 2009.

\section{Mayara Ramos \\ mayara.ramos@gmail.com}

Alexandre Amorim de Reis alexandre.reis@pq.cnpq.br 